\title{
Novel Sesquiterpenes from Alpinia intermedia GAGNEP.
}

\author{
Hideji ITOKAWA, ${ }^{*, a}$ Hiroshi Morita, ${ }^{a}$ TAKeShI KoBAyASHI ${ }^{a}$ \\ Kinzo Watanabe, ${ }^{a}$ and Yoichi IItAKA ${ }^{b}$ \\ Tokyo College of Pharmacy, ${ }^{a}$ Horinouchi 1432-1, Hachioji, Tokyo 192-03, \\ Japan and Faculty of Pharmaceutical Science, ${ }^{b}$ University of Tokyo, \\ Bunkyo-ku, Tokyo 113, Japan
}

(Received December 3, 1986)

\begin{abstract}
Three novel sesquiterpene peroxides, hanalpinol peroxide (I), isohanalpinol (II), and aokumanol (III), a new secoguaiane-type sesquiterpene, epialpinolide (IV), and a new elemophilane-type sesquiterpene, $\Delta^{11(12)}$-eremophilen-10 $\beta$-ol $(\mathrm{V})$, have been isolated from the rhizomes of Alpinia intermedia together with eight known sesquiterpenes, hanalpinol (VI), hanalpinone (VII), isohanalpinone (VIII), alpinenone (IX), furopelargone B (X), furopelargone A (XI), intermedeol (XII) and $\beta$-selinene (XIII). The structures of $\mathrm{I}-\mathrm{V}$ were determined by spectroscopic methods, chemical conversion, and X-ray analysis. Their biosynthetic relationships are discussed.

From the point of view of chemotaxonomy, it is suggested that Alpinia intermedia and Alpinia japonica are closely related plants.
\end{abstract}

Keywords_-Alpinia intermedia; Zingiberaceae; sesquiterpene; guaiane; cyclic peroxide; X-ray analysis; eremophilane; secoguaiane; chemotaxonomy; biosynthesis

In our continuing work on the constituents of Alpinia spp. (Zingiberaceae), ${ }^{1)}$ we have investigated Alpinia intermedia. Seeds of $A$. intermedia have been used as an aromatic stomachic in the same way as Amomi Semen. ${ }^{2)}$ In our previous studies, many novel sesquiterpene peroxides ${ }^{3)}$ have been isolated from Alpinia japonica, which belongs to the same genus. This time, separation of the $n$-hexane- and chloroform-soluble fractions obtained from the rhizomes of $A$. intermedia permitted us to isolate five new sesquiterpenes $(\mathrm{I}-\mathrm{V})$ together with eight known sesquiterpenes (VI-XIII). This paper describes the structure determination of the new sesquiterpenes and discusses the structural relationships between them. The sesquiterpenes obtained from $A$. intermedia are compared with those from $A$. japonica.

Compounds I-III were found to be sesquiterpene peroxides because they were positive to the color reactions for peroxides. ${ }^{4)}$

Compound I, named hanalpinol peroxide, was obtained as a colorless oil having the molecular formula $\mathrm{C}_{15} \mathrm{H}_{24} \mathrm{O}_{4}$, based on chemical ionization mass spectrometry (MS) and high-resolution MS. The proton nuclear magnetic resonance $\left({ }^{1} \mathrm{H}-\mathrm{NMR}\right)$ and carbon- 13 nuclear magnetic resonance $\left({ }^{13} \mathrm{C}-\mathrm{NMR}\right)$ data are shown in Tables $\mathrm{I}$ and II. All the spectroscopic data of I were similar to those of hanalpinol (VI). ${ }^{3 a)}$ The stereochemistry of the cyclic peroxide was suggested to be $\beta$, as in the case of VI, from the coupling constant $(5.3 \mathrm{~Hz})$ between $\mathrm{H}-5$ and $\mathrm{H}-6$. The infrared (IR) absorption band at $3560 \mathrm{~cm}^{-1}$ showed the presence of either a hydroxyl group or a hydroperoxide with intramolecular hydrogen bonding. However, as I gave a stronger peroxide reaction than VI, the four oxygen atoms of I were assumed to belong to two peroxides, one being a cyclic peroxide and the other a hydroperoxide. This assumption was proved to be correct: reduction of I with triphenylphosphine gave a product which was identical with natural hanalpinol (VI). Therefore, the absolute structure of I was determined to be as shown in Chart 1 . The structure and this conversion of I into VI may suggest that I is a biogenetic precursor of VI. 


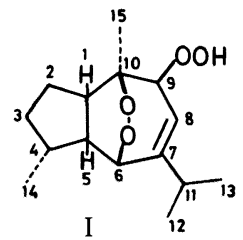<smiles>C=C(C)C1CCC2(O)CCCC(C)C2(C)C1</smiles><smiles>CC(C)C1=CC(=O)[C@]2(C)O[C@]1(O)[C@H]1C(C)CC[C@H]12</smiles>
IX

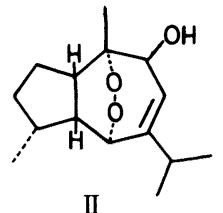<smiles>CC(C)C1=CC(O)C2(C)OC1C1CCC(C)C12</smiles><smiles>[X]C(C)c1ccoc1[C@H]1[C@H](C)CC[C@H]1C(C)=O</smiles>
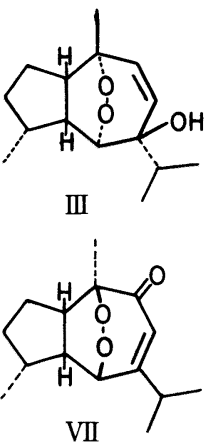<smiles>CC(=O)[C@H]1CCC(C)C1C1OC(=O)C=C1C(C)C</smiles><smiles></smiles><smiles>CC(=O)C1CCC(C)C1c1occc1C(C)C</smiles><smiles>C=C(C)C1CCC2(C)CCCC(C)(O)C2C1</smiles>

XII<smiles>C=C(C)C1CCC2(C)CCCC(=C)C2C1</smiles>

XIII

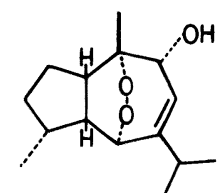

XIV<smiles>C=C(C)C1CC=C2CCCC(C)C2(C)C1</smiles>

XV<smiles>CC1CCCC2=CCC(C(C)(C)O)CC21C</smiles>

$\Delta^{9(10)}$-eremophilen-11-ol

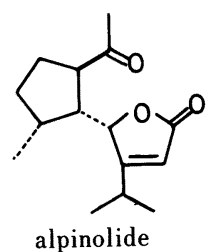

alpinolide

Chart 1

Compound II, named isohanalpinol, was obtained as colorless needles ( $\mathrm{mp} 67^{\circ} \mathrm{C}$ ). The molecular formula, $\mathrm{C}_{15} \mathrm{H}_{24} \mathrm{O}_{3}$ was established by high-resolution MS. The IR spectrum $\left(3620 \mathrm{~cm}^{-1}\right)$ and the positive color reactions for peroxides showed that the three oxygen atoms belonged to a hydroxyl group and a peroxide. The ${ }^{13} \mathrm{C}-\mathrm{NMR}$ spectrum (Table II) suggested the presence of three oxygen-linked carbons, one being hydroxyl group-linked and two being cyclic peroxide-linked $\left(\delta 76.6,77.7\right.$ and 82.3). The ${ }^{1} \mathrm{H}-\mathrm{NMR}$ spectrum (Table I) showed signals ascribable to four methyl groups, that is, two belonging to an isopropyl group attached to an olefin $(\delta 1.06,6 \mathrm{H})$, one doublet methyl group $(\delta 1.11)$ and one methyl group attached to an oxygen atom $(\delta 1.30)$. Compound II was shown to possess the same plane structure as VI. Furthermore, the stereochemistry of the peroxide was deduced to be $\alpha$, as in isohanalpinone (VIII), ${ }^{3 c}$ because of the lack of vicinal coupling between H-5 and H-6. For confirmation of the structure, chemical conversion of VIII into II was carried out: VIII was reduced with sodium borohydride to give two products which were epimers of a hydroxyl moiety. Compound II was identical with the main product. The configuration of the hydroxyl group of II was determined to be $\beta$, because the minor product (XIV) showed the presence of intramolecular hydrogen bonding in the IR spectrum.

Compound III, named aokumanol, was obtained as colorless needles (mp 118.0 $119.0^{\circ} \mathrm{C}$ ) having the molecular formula $\mathrm{C}_{15} \mathrm{H}_{24} \mathrm{O}_{3}$, based on high-resolution MS. The ${ }^{13} \mathrm{C}$ NMR spectrum showed three signals of carbon atoms bearing an oxygen function $(\delta 79.3$, 79.8 and 80.3$)$. Its color reaction for peroxides was positive and the IR spectrum $\left(3620 \mathrm{~cm}^{-1}\right)$ suggested the presence of a hydroxyl group. Therefore the three oxygen atoms were revealed to belong to a peroxide and a hydroxyl group. The double irradiation experiment in ${ }^{1} \mathrm{H}-\mathrm{NMR}$ and the proton correlation spectrum showed two olefinic protons $(\delta 5.59$ and 5.71$)$ with the coupling constant of $10.7 \mathrm{~Hz}$. Hence, the double bond in III was concluded to be cis. Further, 
TABLE I. ${ }^{1} \mathrm{H}-\mathrm{NMR}$ Data for I-IV $\left(400 \mathrm{MHz}\right.$ in $\left.\mathrm{CDCl}_{3}\right)$

\begin{tabular}{cllll}
\hline \hline Proton & \multicolumn{1}{c}{ I } & \multicolumn{1}{c}{ II } & \multicolumn{1}{c}{ III } & \multicolumn{1}{c}{ IV } \\
\hline 1 & $2.67, \mathrm{ddd}$ & $2.73, \mathrm{ddd}$ & $2.45, \mathrm{ddd}$ & $3.06, \mathrm{dt}$ \\
4 & $2.20, \mathrm{~m}$ & & $2.17, \mathrm{~m}$ & $2.27, \mathrm{~m}$ \\
5 & $2.85, \mathrm{dt}$ & & $2.26, \mathrm{t}$ & $2.40, \mathrm{dt}$ \\
6 & $4.44, \mathrm{dd}$ & $4.40, \mathrm{~d}$ & $4.21, \mathrm{~d}$ & $5.59, \mathrm{dd}$ \\
8 & $5.75, \mathrm{dt}$ & $5.55, \mathrm{dt}$ & $5.59, \mathrm{dd}$ & $5.76, \mathrm{t}$ \\
9 & $4.63, \mathrm{dd}$ & $4.17, \mathrm{~d}$ & $5.71, \mathrm{~d}$ & \\
11 & $2.31, \mathrm{sept} . \mathrm{d}$ & $2.37, \mathrm{sept} . \mathrm{d}$ & $2.36, \mathrm{sept}$ & $2.54, \mathrm{sept} . \mathrm{d}$ \\
12 & $1.12, \mathrm{~d}$ & $1.06, \mathrm{~d}$ & $1.01, \mathrm{~d}$ & $1.17, \mathrm{~d}$ \\
13 & $1.16, \mathrm{~d}$ & $1.03, \mathrm{~d}$ & $1.27, \mathrm{~d}$ \\
14 & $1.08, \mathrm{~d}$ & $1.11, \mathrm{~d}$ & $1.02, \mathrm{~d}$ & $0.94, \mathrm{~d}$ \\
15 & $1.46, \mathrm{~s}$ & $1.30, \mathrm{~s}$ & $1.23, \mathrm{~s}$ & $2.23, \mathrm{~s}$ \\
$-\mathrm{OOH}$ & $8.82, \mathrm{br}$ & & & \\
\hline
\end{tabular}

Coupling constants in Hz. I: $1,2=4.6 ; 1,2=11.5 ; 1,5=9.0 ; 4,5=9.0 ; 4,14=7.2 ; 5,6=5.3 ; 6,8=1.3$; $8,9=4.6 ; 8,11=1.3 ; 9, \mathrm{OOH}=1.3 ; 11,12=6.7 ; 11,13=6.7$. II: $1,2=6.5 ; 1,2=9.5 ; 1,5=8.0 ; 4,5=8.0$; $4,14=7.6 ; 6,8=1.2 ; 8,9=10.7 ; 8,11=1.2 ; 11,12=6.9 ; 11,13=6.9$. III: $1,2=6.5 ; 1,2=9.5 ; 1,5=8.0 ; 4,5=$ $8.0 ; 4,14=7.6 ; 6,8=1.8 ; 8,9=10.7 ; 11,12=6.9 ; 11,13=6.9$. IV: $1,2=9.4 ; 1,2=9.4 ; 1,5=7.0 ; 4,5=7.0$; $4,14=7.0 ; 5,6=4.7 ; 6,8=1.5 ; 8,11=1.5 ; 11,12=7.0 ; 11,13=7.0$.

TABLE II. ${ }^{13} \mathrm{C}-\mathrm{NMR}$ Data for I-IV $\left(100 \mathrm{MHz}\right.$ in $\left.\mathrm{CDCl}_{3}\right)$

\begin{tabular}{rcccc}
\hline \hline No. & I & II & III & IV \\
\hline 1 & $44.5(\mathrm{~d})$ & $38.4(\mathrm{~d})$ & $39.8(\mathrm{~d})$ & $55.4(\mathrm{~d})$ \\
2 & $33.2(\mathrm{t})$ & $32.5(\mathrm{t})$ & $33.6(\mathrm{t})$ & $32.3(\mathrm{t})$ \\
3 & $26.2(\mathrm{t})$ & $26.2(\mathrm{t})$ & $24.9(\mathrm{t})$ & $27.3(\mathrm{t})$ \\
4 & $37.5(\mathrm{~d})$ & $37.1(\mathrm{~d})$ & $37.9(\mathrm{~d})$ & $37.7(\mathrm{~d})$ \\
5 & $45.9(\mathrm{~d})$ & $44.9(\mathrm{~d})$ & $44.5(\mathrm{~d})$ & $46.0(\mathrm{~d})$ \\
6 & $85.3(\mathrm{~d})^{a)}$ & $77.7(\mathrm{~d})^{a)}$ & $79.3(\mathrm{~d})$ & $82.6(\mathrm{~d})$ \\
7 & $154.5(\mathrm{~s})$ & $150.0(\mathrm{~s})$ & $\left.79.8(\mathrm{~s})^{a}\right)$ & $173.4(\mathrm{~s})$ \\
8 & $120.9(\mathrm{~d})$ & $124.4(\mathrm{~d})$ & $135.4(\mathrm{~d})^{b)}$ & $114.3(\mathrm{~d})$ \\
9 & $\left.80.6(\mathrm{~d})^{a}\right)$ & $76.6(\mathrm{~d})^{a)}$ & $136.4(\mathrm{~d})^{b}$ & $180.7(\mathrm{~s})$ \\
10 & $84.7(\mathrm{~s})$ & $82.3(\mathrm{~s})$ & $80.3(\mathrm{~s})^{a}$ & $210.5(\mathrm{~s})$ \\
11 & $33.9(\mathrm{~d})$ & $34.8(\mathrm{~d})$ & $34.0(\mathrm{~d})$ & $28.3(\mathrm{~d})$ \\
12 & $20.4(\mathrm{q})$ & $20.9(\mathrm{q})$ & $16.3(\mathrm{q})$ & $20.5(\mathrm{q})$ \\
13 & $23.2(\mathrm{q})$ & $21.0(\mathrm{q})$ & $16.8(\mathrm{q})$ & $22.3(\mathrm{q})$ \\
14 & $15.3(\mathrm{q})$ & $15.4(\mathrm{q})$ & $15.1(\mathrm{q})$ & $16.1(\mathrm{q})$ \\
15 & $25.7(\mathrm{q})$ & $24.3(\mathrm{q})$ & $24.8(\mathrm{q})$ & $30.0(\mathrm{q})$ \\
\hline
\end{tabular}

$a, b)$ Tentative.

the signal at $\delta 5.59$ showed a long-range coupling $(J=1.8 \mathrm{~Hz})$ with the proton $(\delta 4.21)$ linked to an oxygen atom-bearing carbon. The signal at $\delta 4.21$ can not be that of the proton linked to the hydroxyl group-bearing carbon but must be that of the proton linked to a cyclic peroxidebearing carbon because its chemical shift did not change after the addition of trichloroacetyl isocyanate (TIA) ${ }^{5}$ : the hydroxyl group must be tertiary. The spectral investigation and comparison of the IR and NMR data of III with those of known sesquiterpene peroxides (Tables I and II) suggested that III has a guaiane skeleton. The stereochemistry of the cyclic peroxide was considered to be $\alpha$ because of the lack of vicinal coupling between H-5 and H-6. However, to elucidate the whole structure including stereochemical configuration, X-ray analysis of III was undertaken.

Aokumanol (III) was crystallized from $n$-hexane-ethyl acetate solutions as colorless fine 
needles elongated along the $c$ axis. The lattice constants and intensity data were measured on a Philips PW1 100 diffractometer using graphite-monochromated $\mathrm{Cu} K_{\alpha}$ radiation. The size of the crystal used was about $0.02 \times 0.1 \times 0.15 \mathrm{~mm}$. Crystal data are: orthorhombic, space group $P 2_{1} 2_{1} 2_{1}, Z=4, D_{\text {calc }}=1.161 \mathrm{~g} \mathrm{~cm}^{-3}, a=14.608(8), b=17.592(8), c=5.617(4) \AA, U=1443 \AA^{3}$. Intensities of 1330 reflections were measured as above the $2 \sigma(I)$ level out of 1755 in the $2 \theta$ range of $6^{\circ}$ through $178^{\circ}$. The crystal was deteriorated by X-ray irradiation. The intensities of the three standard reflections, 902, 080 and $0 \overline{3} 4$, were monitored every $2 \mathrm{~h}$ and it was found that the extents of decrease of the intensities during the $2 \mathrm{~h}$ intervals were $6.1,20.5$ and $4.5 \%$, respectively, throughout the measurement. The intensities were, therefore, corrected under the simple assumption that they decreased uniformly with respect to time and the direction of the Bragg reflection. The crystal structure was determined by the direct method and refined by the block-diagonal-matrix least-squares method. The $R$ value could not be reduced beyond 0.147 . The final difference electron-density map showed 8 small peaks having peak height greater than $0.35 e / \AA^{3}$, with the maximum value $0.5 e / \AA^{3}$. Some of them were assigned as hydrogen atoms. Presumably errors arising from the crystal deterioration and inadequate correction of the intensities prevented further refinement. The molecular structure having the cis-linked guaiane skeleton, the bond lengths and the bond angles are shown in Fig. 1 and Tables $\mathrm{V}$ and VI. No abnormal features were found in the structure. Hydrogen atoms were located by calculation assuming $s p^{3}$ or $s p^{2}$ configuration about the carbon atoms and their atomic parameters were refined by the least-squares method. The relative configuration of the cyclic peroxide was concluded to be $\alpha$ estimated from the lack of vicinal coupling in the ${ }^{1} \mathrm{H}-\mathrm{NMR}$ spectrum.

Compound IV, a colorless oil, was a secoguaiane-type sesquiterpene named epialpinolide. The molecular formula, $\mathrm{C}_{15} \mathrm{H}_{22} \mathrm{O}_{3}$, was established by high-resolution MS. The IR, ultraviolet (UV) and ${ }^{1} \mathrm{H}-\mathrm{NMR}$ spectra indicated the presence of an unsaturated butenolide (1760 and $\left.1630 \mathrm{~cm}^{-1} ; 214 \mathrm{~nm}, \varepsilon 10900\right)$ and a methyl ketone group $\left(1700 \mathrm{~cm}^{-1} ; \delta 2.23,3 \mathrm{H}, \mathrm{s}\right)$. The above spectral data, ${ }^{13} \mathrm{C}$-NMR spectrum (Table II) and the coupling relationships observed in a detailed proton decoupling experiment were in reasonable agreement with those of alpinolide, ${ }^{6)}$ which has been isolated from Alpinia japonica. The whole structure including the absolute configuration was determined by chemically converting IV into alpinolide by treatment with $p$-toluenesulfonic acid; the spectral data and specific rotation of the product were identical with those of natural alpinolide. Consequently, compound IV was identified as an epimer of alpinolide and the stereochemistry of the methyl ketone group of IV should be $\alpha$. This was supported by a proton nuclear Overhauser effect (NOE) experiment, which showed an NOE of $4.1 \%$ between $\mathrm{H}-1$ and H-5 in IV. On the other hand, no NOE was observed between the corresponding protons in alpinolide.

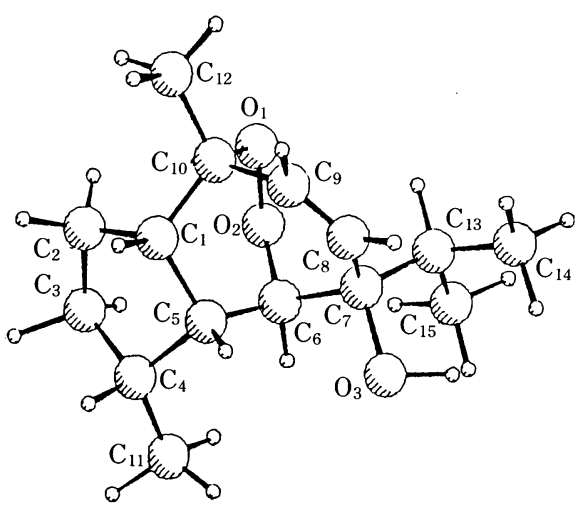

Fig. 1. Molecular Structure Drawn by the PLUTO $^{11)}$ Program 
TABLE III. Atomic Coordinates and Equivalent Isotropic Temperature Factors

\begin{tabular}{|c|c|c|c|c|c|}
\hline No. & Atom & $x 10^{4}$ & $y 10^{4}$ & $z 10^{4}$ & $B_{\text {eq }}\left(\AA^{2}\right)$ \\
\hline 1 & $\mathrm{Cl}$ & $8563(10)$ & $3987 \quad(7)$ & $3997(23)$ & $3.9(0.2)$ \\
\hline 2 & $\mathrm{C} 2$ & $9134(11)$ & $4530(10)$ & $5694(22)$ & $5.4(0.3)$ \\
\hline 3 & $\mathrm{C} 3$ & $10146(12)$ & $4363(13)$ & $5214(23)$ & $7.1(0.3)$ \\
\hline 4 & $\mathrm{C} 4$ & $10137(10)$ & 3964 (9) & $2659(22)$ & $4.8(0.2)$ \\
\hline 5 & $\mathrm{C} 5$ & $9248 \quad(9)$ & 3440 & $2733(17)$ & $3.2(0.2)$ \\
\hline 6 & C6 & $9292 \quad$ (9) & $2705 \quad(7)$ & 3945 (15) & $3.4(0.2)$ \\
\hline 7 & $\mathrm{C} 7$ & $8648 \quad(9)$ & $2077 \quad(8)$ & 3053 (19) & $3.8(0.2)$ \\
\hline 8 & $\mathrm{C} 8$ & 7723 & $2382 \quad(8)$ & $2431(21)$ & $3.8(0.2)$ \\
\hline 9 & C9 & 7358 & $2978 \quad(9)$ & $3279(23)$ & $4.4(0.2)$ \\
\hline 10 & $\mathrm{C} 10$ & 7798 (10) & 3542 (8) & $5219(27)$ & $4.6(0.2)$ \\
\hline 11 & $\mathrm{C} 11$ & $11021(11)$ & $3510(11)$ & 2081 (29) & $6.4(0.3)$ \\
\hline 12 & $\mathrm{C} 12$ & 7099 (11) & 4032 (12) & $6513(30)$ & $7.5(0.4)$ \\
\hline 13 & $\mathrm{C} 13$ & $8577(10)$ & 1364 (8) & $4577(21)$ & $4.4(0.2)$ \\
\hline 14 & $\mathrm{C} 14$ & $7987(12)$ & $738(10)$ & 3548 (29) & $5.9(0.3)$ \\
\hline 15 & $\mathrm{C} 15$ & $9535(11)$ & $1017(11)$ & $5205(26)$ & $6.0(0.3)$ \\
\hline 16 & $\mathrm{Ol}$ & $8197 \quad(6)$ & 3026 & 7043 (12) & $5.0(0.2)$ \\
\hline 17 & $\mathrm{O} 2$ & $9162 \quad(6)$ & 2809 (6) & $6578(11)$ & $4.3(0.2)$ \\
\hline 18 & $\mathrm{O} 3$ & $9097 \quad$ (7) & $1835 \quad(5)$ & 817 (12) & $4.4(0.1)$ \\
\hline No. & Atom & $x 10^{3}$ & $y 10^{3}$ & $z 10^{3}$ & $B_{\text {eq }}\left(\AA^{2}\right)$ \\
\hline 19 & $\mathrm{HCl}$ & $824 \quad(9)$ & $445 \quad(7)$ & $265(28)$ & 7. (4.) \\
\hline 20 & $\mathrm{HC} 2$ & $896 \quad(8)$ & $515 \quad(6)$ & $534(24)$ & 5. (3.) \\
\hline 21 & $\mathrm{H}^{\prime} \mathrm{C} 2$ & 891 (12) & $444(10)$ & $723(41)$ & 13. (6.) \\
\hline 22 & $\mathrm{HC} 3$ & $1041(10)$ & $396 \quad(9)$ & $629(32)$ & 9. (5.) \\
\hline 23 & $\mathrm{H}^{\prime} \mathrm{C} 3$ & 1059 & $496 \quad(6)$ & $514(21)$ & 3. (3.) \\
\hline 24 & $\mathrm{HC} 4$ & 1007 & $446 \quad(6)$ & $122(21)$ & 4. (3.) \\
\hline 25 & HC5 & 904 (11) & $334 \quad(9)$ & $102(34)$ & 11. (5.) \\
\hline 26 & HC6 & 1002 & $252 \quad(5)$ & 358 (19) & 3. (2.) \\
\hline 27 & $\mathrm{HC} 8$ & $737(10)$ & $204 \quad(8)$ & $144(30)$ & 9. (5.) \\
\hline 28 & HC9 & $670 \quad(7)$ & $316 \quad(6)$ & $284(23)$ & 4. (3.) \\
\hline 29 & $\mathrm{HCll}$ & 1098 (13) & $313(10)$ & $73(40)$ & 13. (6.) \\
\hline 30 & $\mathrm{H}^{\prime} \mathrm{C} 11$ & $1163(10)$ & $394 \quad(8)$ & $206(33)$ & 9. (5.) \\
\hline 31 & $\mathrm{H}^{\prime \prime} \mathrm{C} 11$ & $1112(11)$ & $303(10)$ & $329(37)$ & 11. (5.) \\
\hline 32 & $\mathrm{HC} 12$ & 743 (10) & $441 \quad(8)$ & $788(31)$ & 9. (5.) \\
\hline 33 & $\mathrm{H}^{\prime} \mathrm{C} 12$ & $678(10)$ & $448 \quad(8)$ & $529(29)$ & 8. (4.) \\
\hline 34 & $\mathrm{H}^{\prime \prime} \mathrm{C} 12$ & $657 \quad(9)$ & $369 \quad(7)$ & $719(28)$ & 7. (4.) \\
\hline 35 & $\mathrm{HC} 13$ & 824 (12) & 153 (9) & $626(35)$ & 11. (6.) \\
\hline 36 & $\mathrm{HCl} 14$ & 827 (11) & $54 \quad(9)$ & $185(34)$ & 11. (5.) \\
\hline 37 & $\mathrm{H}^{\prime} \mathrm{C} 14$ & $797 \quad(9)$ & $22(7)$ & $468(27)$ & 7. (4.) \\
\hline 38 & $\mathrm{H}^{\prime \prime} \mathrm{C} 14$ & $730 \quad(9)$ & 94 (7) & $325(27)$ & 6. (4.) \\
\hline 39 & $\mathrm{HC} 15$ & $994 \quad(9)$ & $145 \quad(7)$ & $608(24)$ & 6. (3.) \\
\hline 40 & $\mathrm{H}^{\prime} \mathrm{C} 15$ & $946 \quad(8)$ & $47 \quad(7)$ & $624(24)$ & 5. (3.) \\
\hline 41 & $\mathrm{H}^{\prime \prime} \mathrm{C} 15$ & 991 (12) & $83(10)$ & $364(36)$ & 13. (6.) \\
\hline 42 & $\mathrm{HO} 3$ & $898 \quad(7)$ & $122(6)$ & $79(20)$ & 3. (2.) \\
\hline
\end{tabular}

Equivalent positions: $\begin{array}{rrr}x & y & z \\ 1 / 2-x & -y & 1 / 2+z \\ 1 / 2+x & 1 / 2-y & -z \\ -x & 1 / 2+y & 1 / 2-z\end{array}$

Compound $\mathrm{V}$, a colorless oil having the molecular formula $\mathrm{C}_{15} \mathrm{H}_{26} \mathrm{O}$, was an eremophilane-type sesquiterpene. ${ }^{1} \mathrm{H}-\mathrm{NMR}(\delta 1.73,3 \mathrm{H}$, br s; $4.68,4.70$, each $1 \mathrm{H}$, br s) and IR $\left(3080\right.$ and $\left.890 \mathrm{~cm}^{-1}\right)$ spectra indicated the presence of an isopropenyl group. The ${ }^{1} \mathrm{H}-\mathrm{NMR}$ 
TABLE IV. Temperature Factors

\begin{tabular}{|c|c|c|c|c|c|c|c|}
\hline \multirow{3}{*}{$\begin{array}{c}\text { No. } \\
1\end{array}$} & \multirow{3}{*}{$\begin{array}{c}\text { Atom } \\
\mathrm{Cl}\end{array}$} & \multicolumn{6}{|c|}{$U(i j)$ 's are multiplied by $10^{3}$} \\
\hline & & $U_{11}$ & $U_{22}$ & $U_{33}$ & $U_{12}$ & $U_{13}$ & $U_{23}$ \\
\hline & & $73(9)$ & $42(6)$ & 33 (7) & $-2(7)$ & $-6(7)$ & $4(6)$ \\
\hline 2 & $\mathrm{C} 2$ & $79(10)$ & $102(12)$ & $23(6)$ & $-6(10)$ & $-9(8)$ & $-26 \quad(8)$ \\
\hline 3 & $\mathrm{C} 3$ & $84(12)$ & $169(18)$ & $18 \quad(7)$ & $-22(13)$ & $-8(8)$ & $-33(10)$ \\
\hline 4 & $\mathrm{C} 4$ & 71 (9) & $96(11)$ & $14(6)$ & $-5 \quad(9)$ & $-4(6)$ & $-16(7)$ \\
\hline 5 & $\mathrm{C} 5$ & $61 \quad(7)$ & $53(7)$ & $5(4)$ & 5 (7) & $-2(6)$ & $3(5)$ \\
\hline 6 & C6 & $60 \quad(7)$ & $76 \quad(8)$ & $-6(4)$ & 0 & $5(5)$ & $-9 \quad(5)$ \\
\hline 7 & $\mathrm{C} 7$ & 58 & 74 (9) & $12(5)$ & $7(7)$ & $9(6)$ & $-21 \quad(6)$ \\
\hline 8 & $\mathrm{C} 8$ & $58 \quad(8)$ & $69 \quad(8)$ & $17 \quad(5)$ & $8(7)$ & $-8(6)$ & $-4 \quad(6)$ \\
\hline 9 & C9 & $56 \quad(8)$ & $81(10)$ & 32 (7) & $-7 \quad(8)$ & $-15(7)$ & 10 \\
\hline 10 & $\mathrm{C} 10$ & $69 \quad(9)$ & $47 \quad(7)$ & $58 \quad(9)$ & 14 & $-7(8)$ & $-9 \quad(8)$ \\
\hline 11 & $\mathrm{C} 11$ & $61 \quad(9)$ & $138(15)$ & $46 \quad(8)$ & $-13(11)$ & $5(8)$ & $-12(11)$ \\
\hline 12 & $\mathrm{C} 12$ & $67(11)$ & $167(20)$ & $51(10)$ & $45(12)$ & $7(9)$ & $-23(12)$ \\
\hline 13 & $\mathrm{C} 13$ & $83(10)$ & $67 \quad(9)$ & $18 \quad(6)$ & $14 \quad(8)$ & $22(7)$ & $10 \quad(6)$ \\
\hline 14 & $\mathrm{C} 14$ & $93(12)$ & $81(11)$ & $48 \quad(9)$ & $-25(10)$ & $16(9)$ & -11 \\
\hline 15 & $\mathrm{C} 15$ & $74(10)$ & $123(14)$ & 31 & $14(10)$ & $6(8)$ & $9(10)$ \\
\hline 16 & $\mathrm{Ol}$ & $67 \quad(6)$ & $123 \quad(8)$ & -1 (3) & 21 & $0(4)$ & $10 \quad(5)$ \\
\hline 17 & $\mathrm{O} 2$ & $59 \quad(5)$ & $120 \quad(8)$ & -14 & $9(6)$ & $-9(3)$ & -1 (4) \\
\hline 18 & $\mathrm{O} 3$ & $82 \quad(6)$ & $79 \quad(6)$ & $6 \quad(3)$ & $2(6)$ & $7(4)$ & -12 (4) \\
\hline No. & Atom & $U(i j)$ 's & $\begin{array}{l}\text { multiplied by } 10^{2} \\
U_{11}\end{array}$ & No. & Atom & $U(i j)$ 's are & plied by $10^{2}$ \\
\hline 19 & $\mathrm{HCl}$ & & $9(5)$ & 31 & $\mathrm{H}^{\prime \prime} \mathrm{C} 11$ & & \\
\hline 20 & $\mathrm{HC} 2$ & & $6(4)$ & 32 & $\mathrm{HCl} 12$ & & \\
\hline 21 & $\mathrm{H}^{\prime} \mathrm{C} 2$ & & $16(8)$ & 33 & $\mathrm{H}^{\prime} \mathrm{C} 12$ & & \\
\hline 22 & $\mathrm{HC} 3$ & & $11(6)$ & 34 & $\mathrm{H}^{\prime \prime} \mathrm{C} 12$ & & \\
\hline 23 & $\mathrm{H}^{\prime} \mathrm{C} 3$ & & $4(3)$ & 35 & $\mathrm{HC} 13$ & & \\
\hline 24 & $\mathrm{HC} 4$ & & $5(4)$ & 36 & $\mathrm{HCl} 4$ & & \\
\hline 25 & $\mathrm{HC} 5$ & & $14(7)$ & 37 & $\mathrm{H}^{\prime} \mathrm{Cl} 14$ & & \\
\hline 26 & HC6 & & $4(3)$ & 38 & $\mathrm{H}^{\prime \prime} \mathrm{C} 14$ & & \\
\hline 27 & $\mathrm{HC} 8$ & & $11(6)$ & 39 & $\mathrm{HC} 15$ & & \\
\hline 28 & HC9 & & $5(4)$ & 40 & $\mathrm{H}^{\prime} \mathrm{C} 15$ & & \\
\hline 29 & $\mathrm{HCll}$ & & $17(8)$ & 41 & $\mathrm{H}^{\prime \prime} \mathrm{C} 15$ & & \\
\hline 30 & $\mathrm{H}^{\prime} \mathrm{Cl} 1$ & & $11(6)$ & 42 & $\mathrm{HO} 3$ & & \\
\hline
\end{tabular}

Temperature factor $T$ is in the form of

$$
T=\exp \left(-2 \pi^{2}\left(U_{11} H^{2} A^{* 2}+U_{22} K^{2} B^{* 2}+U_{33} L^{2} C^{* 2}+2 U_{12} H K A^{*} B^{*}+2 U_{13} H L A^{*} C^{*}+2 U_{23} K L B^{*} C^{*}\right)\right) .
$$

spectrum indicated the presence of a singlet methyl $(\delta 0.84)$ and a doublet methyl $(\delta 0.80)$ groups. A tertiary hydroxyl group was indicated by the IR $\left(3630 \mathrm{~cm}^{-1}\right)$ and ${ }^{13} \mathrm{C}-\mathrm{NMR}(\delta 73.2$, s) spectra. These spectral data suggest that compound $V$ is of eudesmane or eremophilane type, possessing a tertiary hydroxyl group. Dehydration of $\mathrm{V}$ gave $\mathrm{XV}$ which was also produced by dehydration of $\Delta^{9(10)}$-eremophilen-11-ol ${ }^{7)}$ isolated from Alpinia japonica. Consequently, $\mathrm{V}$ was concluded to have the eremophilane skeleton and a hydroxyl group at $\mathrm{C}-10$. The configuration of the hydroxyl group was deduced from the pyridine-induced solvent effect ${ }^{8)}$ in the ${ }^{1} \mathrm{H}-\mathrm{NMR}$ spectrum. In the ${ }^{1} \mathrm{H}-\mathrm{NMR}$ spectrum (pyridine- $d_{5}$ ), the singlet methyl group signal showed a downfield shift $(-0.22 \mathrm{ppm})$ owing to the vicinal deshielding effect. Therefore the hydroxyl group at C-10 and the methyl group at C-5 must be syn. That is, the configuration of the hydroxyl group must be $\beta$. Compound $\mathrm{V}$ was determined to be $\Delta^{11(12)}$-eremophilen-10 $\beta$-ol. 
TABLE V. Bond Lengths $(\AA)$

\begin{tabular}{llll}
\hline & Length (S.T.D.) & & Length (S.T.D.) \\
\hline $\mathrm{C} 1-\mathrm{C} 2$ & $1.585(20)$ & $\mathrm{C} 7-\mathrm{C} 8$ & $1.495(18)$ \\
$\mathrm{C} 1-\mathrm{C} 5$ & $1.559(18)$ & $\mathrm{C} 7-\mathrm{C} 13$ & $1.522(18)$ \\
$\mathrm{C} 1-\mathrm{C} 10$ & $1.528(20)$ & $\mathrm{C} 7-\mathrm{O} 3$ & $1.479(14)$ \\
$\mathrm{C} 2-\mathrm{C} 3$ & $1.531(24)$ & $\mathrm{C} 8-\mathrm{C} 9$ & $1.269(20)$ \\
$\mathrm{C} 3-\mathrm{C} 4$ & $1.598(20)$ & $\mathrm{C} 9-\mathrm{C} 10$ & $1.608(20)$ \\
$\mathrm{C} 4-\mathrm{C} 5$ & $1.593(20)$ & $\mathrm{C} 10-\mathrm{C} 12$ & $1.521(23)$ \\
$\mathrm{C} 4-\mathrm{C} 11$ & $1.552(23)$ & $\mathrm{C} 10-\mathrm{O} 1$ & $1.487(17)$ \\
$\mathrm{C} 5-\mathrm{C} 6$ & $1.464(17)$ & $\mathrm{C} 13-\mathrm{C} 14$ & $1.513(22)$ \\
$\mathrm{C} 6-\mathrm{C} 7$ & $1.535(18)$ & $\mathrm{C} 13-\mathrm{C} 15$ & $1.566(22)$ \\
$\mathrm{C} 6-\mathrm{O} 2$ & $1.502(11)$ & $\mathrm{O} 1-\mathrm{O} 2$ & $1.485(13)$ \\
\hline
\end{tabular}

S.T.D.: The standard deviation

TABle VI. Bond Angles ( $\left.{ }^{\circ}\right)$

\begin{tabular}{llll}
\hline \hline & Angle (S.T.D.) & & Angle (S.T.D.) \\
\hline C2-C1-C5 & $108.0(11)$ & $\mathrm{C} 6-\mathrm{C} 7-\mathrm{C} 13$ & $116.8(10)$ \\
$\mathrm{C} 2-\mathrm{C} 1-\mathrm{C} 10$ & $115.0(11)$ & $\mathrm{C} 6-\mathrm{C} 7-\mathrm{O} 3$ & $102.3(9)$ \\
$\mathrm{C} 5-\mathrm{C} 1-\mathrm{C} 10$ & $110.9(11)$ & $\mathrm{C} 13-\mathrm{C} 7-\mathrm{O} 3$ & $105.7(10)$ \\
$\mathrm{C} 3-\mathrm{C} 2-\mathrm{C} 1$ & $106.6(12)$ & $\mathrm{C} 9-\mathrm{C} 8-\mathrm{C} 7$ & $126.1(12)$ \\
$\mathrm{C} 4-\mathrm{C} 3-\mathrm{C} 2$ & $103.6(13)$ & $\mathrm{C} 10-\mathrm{C} 9-\mathrm{C} 8$ & $126.6(13)$ \\
$\mathrm{C} 5-\mathrm{C} 4-\mathrm{C} 3$ & $103.7(11)$ & $\mathrm{C} 12-\mathrm{C} 10-\mathrm{C} 1$ & $114.5(13)$ \\
$\mathrm{C} 5-\mathrm{C} 4-\mathrm{C} 11$ & $112.7(12)$ & $\mathrm{C} 12-\mathrm{C} 10-\mathrm{C} 9$ & $114.0(12)$ \\
$\mathrm{C} 3-\mathrm{C} 4-\mathrm{C} 11$ & $114.0(13)$ & $\mathrm{C} 12-\mathrm{C} 10-\mathrm{O} 1$ & $106.2(12)$ \\
$\mathrm{C} 6-\mathrm{C} 5-\mathrm{C} 1$ & $111.2(10)$ & $\mathrm{C} 1-\mathrm{C} 10-\mathrm{C} 9$ & $107.7(11)$ \\
$\mathrm{C} 6-\mathrm{C} 5-\mathrm{C} 4$ & $119.2(10)$ & $\mathrm{C} 1-\mathrm{C} 10-\mathrm{O} 1$ & $109.6(11)$ \\
$\mathrm{C} 1-\mathrm{C} 5-\mathrm{C} 4$ & $100.2(10)$ & $\mathrm{C} 9-\mathrm{C} 10-\mathrm{O} 1$ & $104.3(10)$ \\
$\mathrm{C} 7-\mathrm{C} 6-\mathrm{C} 5$ & $117.2(10)$ & $\mathrm{C} 14-\mathrm{C} 13-\mathrm{C} 7$ & $115.1(12)$ \\
$\mathrm{C} 7-\mathrm{C} 6-\mathrm{O} 2$ & $109.4(9)$ & $\mathrm{C} 14-\mathrm{C} 13-\mathrm{C} 15$ & $108.2(12)$ \\
$\mathrm{C} 5-\mathrm{C} 6-\mathrm{O} 2$ & $110.2(9)$ & $\mathrm{C} 7-\mathrm{C} 13-\mathrm{C} 15$ & $112.8(11)$ \\
$\mathrm{C} 8-\mathrm{C} 7-\mathrm{C} 6$ & $111.8(10)$ & $\mathrm{O} 2-\mathrm{O} 1-\mathrm{C} 10$ & $114.1(9)$ \\
$\mathrm{C} 8-\mathrm{C} 7-\mathrm{C} 13$ & $111.5(11)$ & $\mathrm{C} 6-\mathrm{O} 2-\mathrm{O} 1$ & $109.0(8)$ \\
$\mathrm{C} 8-\mathrm{C} 7-\mathrm{O} 3$ & $107.8(10)$ & & \\
\hline
\end{tabular}
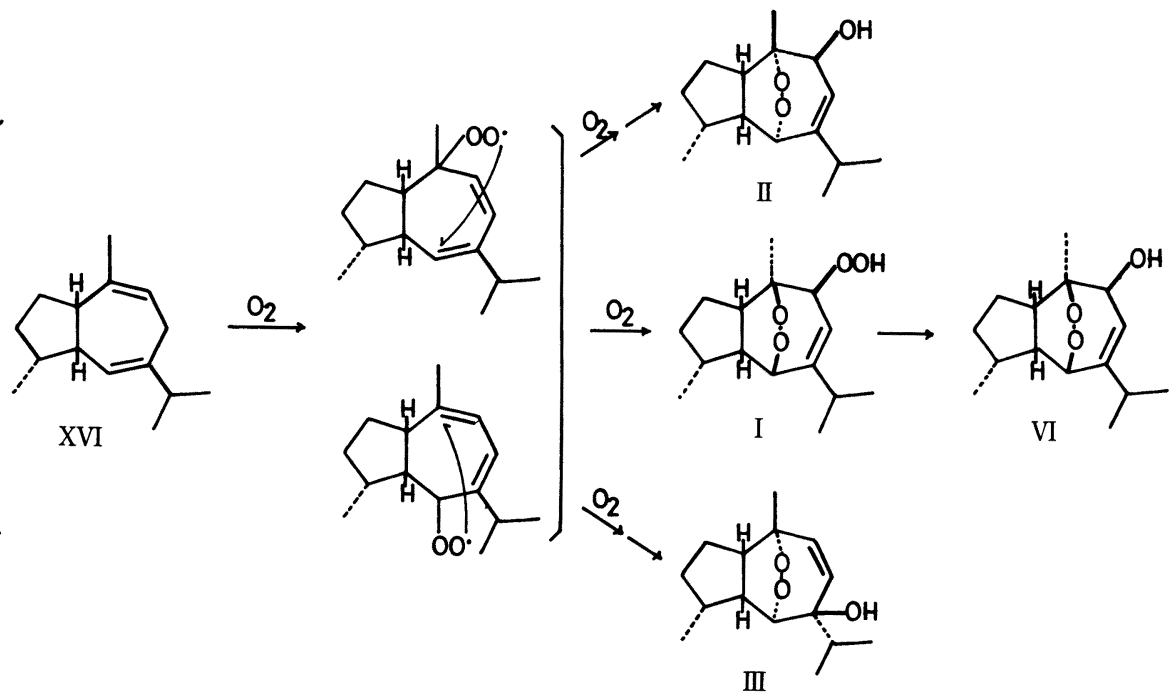

Chart 2. Proposed Biogenetic Pathway of I, II, III and VI 
Eight known sesquiterpenes, hanalpinol (VI), hanalpinone (VII), isohanalpinone (VIII), alpinenone (IX), furopelargone B (X), furopelargone A (XI), intermedeol (XII), ${ }^{9)}$ and $\beta$ selinene (XIII) ${ }^{10)}$ were also isolated. The structures of $\mathrm{I}-\mathrm{V}$ are very similar to those of the above known sesquiterpenes VI-XI which have already been isolated from Alpinia japonica. ${ }^{3)}$ From the chemotaxonomic viewpoint, it is suggested that Alpinia intermedia and Alpinia japonica are closely related plants.

Furthermore, from the biogenetic point of view, I was considered to be an immediate biogenetic precursor of VI, and these sesquiterpene peroxides were assumed to be derived from similar compounds which possess a hydroperoxide moiety at C-9. We propose that these compounds are derived from compound XVI, which has two double bonds at C-6 and C-9, by successive oxidations with oxygen as shown in Chart 2.

\section{Experimental}

All melting points were recorded on a Yanagimoto micro melting point apparatus, and are uncorrected. Spectral data were obtained on the following instruments; optical rotation on a JASCO DIP-4, IR on a JASCO A-302, UV on a Hitachi 557, NMR on a Brucker AM400, and MS on a Hitachi M-80. High-performance liquid chromatography (HPLC) was carried out on a CIG column system (Kusano Scientific Co., Tokyo) with Iatrobeads (60 $\mu$ silica gel, IATRON Co., Tokyo) as the stationary phase.

Extraction and Isolation_-Fresh rhizomes $(14.0 \mathrm{~kg})$ of Alpinia intermedia collected at Nomosaki, Nagasaki, in August 1984 were extracted twice with methanol under an argon atmosphere. The methanol extract was treated with $n$-hexane, and the $n$-hexane layer was concentrated to give a yellow oil $(18.5 \mathrm{~g})$. Then the methanol layer was treated with chloroform, and the chloroform layer was concentrated to give a brown oil $(24.0 \mathrm{~g})$. The $n$-hexane and chloroform extracts were each subjected to column chromatography on silica gel with a linear $n$-hexane-ethyl acetate gradient system and a linear chloroform-methanol gradient system, respectively. Repeated $\mathrm{HPLC}$ and $\mathrm{AgNO}_{3}-$ HPLC of each fraction using an $n$-hexane-ethyl acetate system, an $n$-hexane-chloroform-acetonitrile system, a benzene-ethyl acetate system, and a benzene-chloroform-acetonitrile system gave I (100 mg), II (100 mg), III (50 mg), IV (100 mg), V (100 mg), VI (500 mg), VII (300 mg), VIII (200 mg), IX (100 mg), X (1.5 g), XI (200 mg), XII (100 mg), and XIII $(500 \mathrm{mg})$.

Compound I (Hanalpinol Peroxide): A colorless oil, $[\alpha]_{\mathrm{D}}+135.0^{\circ}\left(c=0.50, \mathrm{CHCl}_{3}\right), \mathrm{CI}-\mathrm{MS} m / z(\%): 269$ $\left(\mathrm{M}^{+}+1,1\right)$; EI-MS $m / z(\%) 250\left(\mathrm{M}^{+}-18,5\right.$, Calcd for $\mathrm{C}_{15} \mathrm{H}_{22} \mathrm{O}_{3}, 250.1559$; Found 250.1569), 219 (5), 191 (10), 147 (20), 95 (60), 81 (100), 71 (65). IR $\left(\mathrm{CCl}_{4}\right) \mathrm{cm}^{-1}: 3560,3480,2970,2880,1465,1390,1380,970,885$.

Compound II (Isohanalpinol): Colorless needles, mp $67.0^{\circ} \mathrm{C},[\alpha]_{\mathrm{D}}-21.0^{\circ}\left(c=0.10, \mathrm{CHCl}_{3}\right) . \mathrm{MS} m / z(\%): 252$ $\left(\mathrm{M}^{+}, 54\right.$, Calcd for $\mathrm{C}_{15} \mathrm{H}_{24} \mathrm{O}_{3}, 252.1724$; Found 252.1694), 235 (100), 201 (40), 175 (54), 149 (12), 95 (12). IR $\left(\mathrm{CCl}_{4}\right) \mathrm{cm}^{-1}: 3620,2960,2940,2880,1460,1385,1005$.

Compound III (Aokumanol): Colorless needles, mp 118.0-119.0 ${ }^{\circ} \mathrm{C},[\alpha]_{\mathrm{D}}+6.7^{\circ}\left(c=0.06, \mathrm{CHCl}_{3}\right) . \mathrm{MS} m / z(\%)$ : $252\left(\mathrm{M}^{+}, 1\right.$, Calcd for $\mathrm{C}_{15} \mathrm{H}_{24} \mathrm{O}_{3}, 252.1724$; Found 252.1740), 234 (10), 191 (15), 163 (18), 141 (23), 123 (100), 81 (37), 71 (32), 55 (35). IR $\left(\mathrm{CCl}_{4}\right) \mathrm{cm}^{-1}: 3620,2960,2880,1465,1455,1380,1370,1135,985,925$.

Compound IV (Epialpinolide): A colorless oil, $[\alpha]_{\mathrm{D}}-7.1^{\circ}\left(c=0.07, \mathrm{CHCl}_{3}\right) . \mathrm{MS} m / z(\%): 250\left(\mathrm{M}^{+}, 100, \mathrm{Calcd}\right.$ for $\mathrm{C}_{15} \mathrm{H}_{22} \mathrm{O}_{3}, 250.1567$; Found 250.1549), 207 (37), 179 (62), 165 (55), 139 (42), 126 (86), 96 (49), 81 (54), 69 (48). IR $\left(\mathrm{CCl}_{4}\right) \mathrm{cm}^{-1}: 2970,2880,1760,1700,1630,1470,1385,1360,1325,1265,1190,1175,1160,1045,1005,970,915,865$. UV (EtOH) nm (E): 214 (10900).

Compound V $\left(\Delta^{11(12)}\right.$-Eremophilen-10 $\beta$-ol): A colorless oil, $[\alpha]_{\mathrm{D}}+29.2^{\circ}\left(c=0.12, \mathrm{CHCl}_{3}\right) . \mathrm{MS} m / z(\%): 222$ $\left(\mathrm{M}^{+}, 6\right.$, Calcd for $\mathrm{C}_{15} \mathrm{H}_{26} \mathrm{O}, 222.1981$; Found 222.1971), 204 (60), 189 (44), 161 (35), 126 (44), 123 (44), 111 (69), 109 (69), $81(74), 69(64), 67(58), 55(100)$. IR $\left(\mathrm{CCl}_{4}\right) \mathrm{cm}^{-1}: 3630,3080,2930,2870,1645,1450,1380,1010,960,890 .{ }^{1} \mathrm{H}-$ NMR $\left(\mathrm{CDCl}_{3}\right) \delta \mathrm{ppm}: 0.80(3 \mathrm{H}, \mathrm{d}, J=6.8 \mathrm{~Hz}), 0.84(3 \mathrm{H}, \mathrm{s}), 1.73(3 \mathrm{H}, \mathrm{br} \mathrm{s}), 4.68(1 \mathrm{H}, \mathrm{br} \mathrm{s}), 4.70(1 \mathrm{H}, \mathrm{br} \mathrm{s}) .{ }^{13} \mathrm{C}-\mathrm{NMR}$ $\left(\mathrm{CDCl}_{3}\right) \delta$ ppm: $15.4(\mathrm{q}), 15.4(\mathrm{q}), 21.0(\mathrm{q}), 23.0(\mathrm{t}), 26.8(\mathrm{t}), 30.2(\mathrm{t}), 32.9(\mathrm{t}), 33.1(\mathrm{~d}), 36.6(\mathrm{t}), 36.8(\mathrm{t}), 38.8(\mathrm{~d}), 41.2(\mathrm{~s})$, $73.2(\mathrm{~s}), 108.2(\mathrm{t}), 150.5(\mathrm{~s})$.

Compounds VI-XI : Compounds VI-XI were identical with those which have been isolated from Alpinia japonica and reported in our previous paper.

Compound XII (Intermedeol): Colorless needles, mp 46.0-47.0 ${ }^{\circ} \mathrm{C},[\alpha]_{\mathrm{D}}+7.1^{\circ}\left(c=0.17, \mathrm{CHCl}_{3}\right)$. $\mathrm{MS} m / z(\%)$ : $222\left(\mathrm{M}^{+}, 4\right), 204$ (90), 189 (70), 161 (50), 135 (40), 123 (51), 109 (58), 95 (62), 81 (100), 71 (82), 67 (60), 55 (60). IR $\left(\mathrm{CCl}_{4}\right) \mathrm{cm}^{-1}: 3625,3085,2940,1640,1450,1385,1220,1170,1090,1065,910,890 .{ }^{1} \mathrm{H}-\mathrm{NMR}\left(\mathrm{CCl}_{4}\right) \delta \mathrm{ppm}: 0.92(3 \mathrm{H}$, s), $1.02(3 \mathrm{H}, \mathrm{s}), 1.73(3 \mathrm{H}, \mathrm{s}), 4.86(2 \mathrm{H}, \mathrm{br} \mathrm{s})$.

Compound XIII $\left(\beta\right.$-Selinene): A colorless oil, $[\alpha]_{\mathrm{D}}+41.9^{\circ}\left(c=0.09, \mathrm{CHCl}_{3}\right) . \mathrm{MS} m / z(\%): 204\left(65, \mathrm{M}^{+}\right), 189$ (37), 175 (20), 161 (53), 147 (39), 133 (42), 122 (70), 105 (100). IR (neat) $\mathrm{cm}^{-1}: 3050,2910,1640,1440,1380,885 .{ }^{1} \mathrm{H}-$ NMR $\left(\mathrm{CDCl}_{3}\right) \delta$ ppm: $0.72(3 \mathrm{H}, \mathrm{s}), 1.76(3 \mathrm{H}, \mathrm{s}), 4.44(1 \mathrm{H}, \mathrm{br} \mathrm{s}), 4.73(3 \mathrm{H}, \mathrm{br} \mathrm{s}) .{ }^{13} \mathrm{C}-\mathrm{NMR}\left(\mathrm{CDCl}_{3}\right) \delta \mathrm{ppm}: 16.4(\mathrm{q})$, 
$21.0(\mathrm{q}), 23.5(\mathrm{t}), 26.9(\mathrm{t}), 29.5(\mathrm{t}), 36.0(\mathrm{~s}), 36.9(\mathrm{t}), 41.2(\mathrm{t}), 42.0(\mathrm{t}), 45.9(\mathrm{~d}), 49.9(\mathrm{~d}), 105.4(\mathrm{t}), 108.2(\mathrm{t}), 150.9(\mathrm{~s})$, $151.1(\mathrm{~s})$.

Reduction of I with Triphenylphosphine to Give VI-An ether solution $(2 \mathrm{ml})$ of I (15 mg) was treated with triphenylphosphine $(25 \mathrm{mg}$ ) for a few minutes at room temperature, then evaporated. The residue was subjected to HPLC ( $n$-hexane : chloroform : acetonitrile $=7: 2.6: 0.5)$ to give VI as a colorless oil ( $13 \mathrm{mg}$ ), whose spectral data (including specific rotation) were identical with those of natural hanalpinol.

Reduction of VIII with Sodium Borohydride to Give II-A methanol solution of VIII $(25 \mathrm{mg})$ was treated with an excess of sodium borohydride. After work-up in the usual way, the product was subjected to HPLC ( $n$-hexane : ethyl acetate $=4: 1)$ to give II as colorless needles $(22.0 \mathrm{mg})$ whose spectral data (including specific rotation) were identical with those of natural isohanalpinol, and XIV as a colorless oil $(1.2 \mathrm{mg}) .{ }^{1} \mathrm{H}-\mathrm{NMR}\left(\mathrm{CDCl}_{3}\right)$ of XIV $\delta \mathrm{ppm}$ : $1.05(6 \mathrm{H}, \mathrm{d}, J=7.0 \mathrm{~Hz}), 1.17(3 \mathrm{H}, \mathrm{d}, J=7.0 \mathrm{~Hz}), 1.24(3 \mathrm{H}, \mathrm{s}), 3.90(1 \mathrm{H}, \mathrm{dd}, J=4.5,11.5 \mathrm{~Hz}), 4.41(1 \mathrm{H}, \mathrm{br} \mathrm{s}), 5.52(1 \mathrm{H}$, ddd, $J=1.5,1.5,4.5 \mathrm{~Hz})$. IR $\left(\mathrm{CCl}_{4}\right)$ of XIV $\mathrm{cm}^{-1}: 3580,2960,2880,1460,1385,1370,1010$.

Conversion of IV to Alpinolide IV $(10 \mathrm{mg})$ was stirred with $p$-toluenesulfonic acid $(20 \mathrm{mg})$ in benzene $(2 \mathrm{ml})$ for a day at room temperature. Then the reaction mixture was filtered, and the filtrate was washed with saturated sodium bicarbonate solution and brine, dried over magnesium sulfate, and evaporated. The product was purified by HPLC (benzene : chloroform : acetonitrile $=5: 3: 1)$ to give alpinolide $(7 \mathrm{mg}$ ), whose spectral data (including specific rotation) were identical with those of natural alpinolide.

Dehydration of $\mathbf{V}$ to Give $\mathbf{X V}$ - Thionyl chloride $(0.2 \mathrm{ml})$ was added to a cooled solution of $\mathrm{V}(30 \mathrm{mg})$ in pyridine $(2 \mathrm{ml})$ and the reaction mixture was left for $1.5 \mathrm{~h}$ at $0{ }^{\circ} \mathrm{C}$. The mixture was poured into ice-water and extracted with ether, and the ether layer was washed with saturated sodium bicarbonate solution and saturated sodium chloride solution, then dried (magnesium sulfate) and evaporated, leaving a colorless oil (20 mg). This was purified by HPLC ( $n$-hexane) to give XV as a colorless oil $(10 \mathrm{mg})$ whose spectral data (including specific rotation) were identical with those of $\Delta^{9(10), 11(12)}$-eremophilene.

Acknowledgement We thank Prof. N. Kawano, Dr. H. Ohashi and Dr. T. Ikenaga, Faculty of Pharmacy, University of Nagasaki, for support to collect the plant material.

\section{References}

1) H. Itokawa, M. Morita, and S. Mihashi, Chem. Pharm. Bull., 28, 3452 (1980); H. Itokawa, H. Morita, I. Midorikawa, R. Aiyama, and M. Morita, ibid., 33, 4889 (1985); M. Kuroyanagi, T. Noro, S. Fukushima, R. Aiyama, A. Ikuta, H. Itokawa, and M. Morita, ibid., 31, 1554 (1983); H. Itokawa, R. Aiyama, and A. Ikuta, Phytochemistry, 21, 241 (1982); H. Itokawa, H. Morita, T. Sumitomo, N. Totsuka, and K. Takeya, Planta Medica, 53, 32 (1987); H. Morita and H. Itokawa, Chem. Lett., 1986, 1205.

2) T. Namba, "Coloured Illustrations of Wakan-Yaku," Hoikusha Publishing Co., Ltd., 1980, p. 246.

3) a) H. Itokawa, K. Watanabe, H. Morita, S. Mihashi, and Y. Iitaka, Chem. Pharm. Bull., 33, 2023 (1985); b) H. Itokawa, H. Morita, and K. Watanabe, ibid., 35, 1460 (1987); c) H. Itokawa, H. Morita, K. Osawa, K. Watanabe, and Y. Iitaka, ibid., 35, 2849 (1987).

4) E. Knappe and D. Peteri, Z. Anal. Chem., 190, 386 (1962).

5) V. W. Goodlett, Anal. Chem., 37, 431 (1965).

6) H. Itokawa, H. Morita, K. Watanabe, A. Takase, and Y. Iitaka, Chem. Lett., 1984, 1687.

7) H. Itokawa, H. Morita, K. Watanabe, S. Mihashi, and Y. Iitaka, Chem. Pharm. Bull., 33, 1148 (1985).

8) P. V. Demarco, E. Farkas, D. Doddrell, B. L. Mylari, and E. Wenkert, J. Am. Chem. Soc., 90, 5480 (1968).

9) G. L. Chetty, V. B. Zalkow, and L. H. Zalkow, Tetrahedron Lett., 1968, 3223; J. W. Huffman and L. H. Zalkow, ibid., 1973, 751 .

10) B. D. Mackenzie, Mario. M. Angelo, and J. Wolinsky, J. Org. Chem., 44, 4042 (1979).

11) Motherwell, W. D. S. (1972), PLUTO. A program for drawing crystal and molecular structures, University Chemical Laboratory, Cambridge, England. 